\title{
A 2-APPROXIMATION ALGORITHM FOR THE MINIMUM KNAPSACK PROBLEM WITH A FORCING GRAPH
}

\author{
Yotaro Takazawa Shinji Mizuno \\ Tokyo Institute of Technology
}

(Received May 30, 2016; Revised October 5, 2016)

Abstract Carnes and Shmoys [2] presented a 2-approximation algorithm for the minimum knapsack problem. We extend their algorithm to the minimum knapsack problem with a forcing graph (MKPFG), which has a forcing constraint for each edge in the graph. The forcing constraint means that at least one item (vertex) of the edge must be packed in the knapsack. The problem is strongly NP-hard, since it includes the vertex cover problem as a special case. Generalizing the proposed algorithm, we also present an approximation algorithm for the covering integer program with 0-1 variables.

Keywords: Combinatorial optimization, approximation algorithms, minimum knapsack problem, forcing graph, covering integer program

\section{Introduction}

The knapsack problem is one of the most fundamental problems in combinatorial optimization. The problems with additional constraints often appear in practice and algorithms for those problems have been studied, see the survey of Wilbaut et al. [14]. In this paper, we design approximation algorithms with guaranteed accuracy for such problems.

For a given minimization problem having an optimal solution, an algorithm is called an $\alpha$-approximation algorithm if it runs in polynomial time and produces a feasible solution whose objective value is less than or equal to $\alpha$ times the optimal value. Carnes and Shmoys [2] presented a 2-approximation algorithm for the following minimum knapsack problem:

$$
\begin{array}{ll}
\min & \sum_{j \in V} c_{j} x_{j} \\
\text { s.t. } & \sum_{j \in V} a_{j} x_{j} \geq b, \\
& x_{j} \in\{0,1\}, \quad \forall j \in V=\{1, \cdots, n\},
\end{array}
$$

where $V$ is a set of $n$ items, $a_{j}, c_{j} \geq 0(j \in V)$, and $b>0$. Without loss of generality, we assume $\sum_{j \in V} a_{j} \geq b$ so that the problem is feasible.

In this paper, we propose a 2-approximation algorithm for the minimum knapsack 
problem with a forcing graph:

$$
\begin{array}{c|ll}
\min & \sum_{j \in V} c_{j} x_{j} \\
\text { s.t. } & \sum_{j \in V} a_{j} x_{j} \geq b, \\
& x_{i}+x_{j} \geq 1, \quad \forall\{i, j\} \in E, \\
& x_{j} \in\{0,1\}, \quad \forall j \in V=\{1, \cdots, n\},
\end{array}
$$

by extending the algorithm of Carnes and Shmoys [2], where $E$ is a set of edges $\{i, j\} \in V \times V$. The constraint $x_{i}+x_{j} \geq 1$ means that either $i$ or $j$ must be chosen. It is called a forcing constraint and the graph $G=(V, E)$ is called a forcing graph, which is recently used not only for the knapsack problem [13] but also for other combinatorial optimization problems such as minimum spanning tree, matching and shortest path problems [4] and the maximum flow problem [12].

The problem MKPFG (1.2) includes the minimum weight vertex cover problem (VCP) as a special case. It is known that VCP is a strongly NP-hard problem and has inapproximability such that the problem is hard to approximate within any constant factor better than 1.36 unless $P=N P$ [5] and 2 under unique games conjecture [9]. It follows that MKPFG is strongly NP-hard and has at least the same inapproximability as VCP. Bar-Yehuda and Even [1] proposed a 2-approximation algorithm for VCP, so we also extend their result.

The maximum version of MKPFG is known as the knapsack problem with a conflict graph (KPCG). KPCG is the maximum knapsack problem with disjunctive constraints for pairs of items which cannot be packed simultaneously in the knapsack. KPCG is also referred to as the disjunctively constrained knapsack problem. Exact and heuristic algorithms for KPCG were studied by [7, 8, 15] and approximation algorithms were proposed by $[11,13]$. Any exact algorithm for KPCG can solve MKPFG since MKPFG can be transformed into KPCG by complementing the variables. However, the approach of converting MKPFG into KPCG cannot be used in general when we consider the performance guarantee of approximation algorithms. To our knowledge, no approximation algorithms for MKPFG are presented so far.

In section 3, we generalize our algorithm to the covering integer program with $0-1$ variables (CIP), which is also referred to as the capacitated covering problem.

\section{An Algorithm and Analysis}

Carnes and Shmoys [2] used the following LP relaxation of the minimum knapsack problem (1.1), which was constructed by Carr et al. [3]:

$$
\begin{array}{lll}
\min & \sum_{j \in V} c_{j} x_{j} & \\
\text { s.t. } & \sum_{\substack{j \in V \backslash A\\
}} a_{j}(A) x_{j} \geq b(A), & \forall A \subseteq V, \\
& x_{j} \geq 0, & \forall j \in V,
\end{array}
$$

where

$$
\begin{aligned}
b(A) & =\max \left\{0, b-\sum_{j \in A} a_{j}\right\}, & & \forall A \subseteq V, \\
a_{j}(A) & =\min \left\{a_{j}, b(A)\right\}, & & \forall A \subseteq V, \forall j \in V \backslash A .
\end{aligned}
$$

It is known that any feasible 0-1 solution of (2.1) is feasible for (1.1). 
Similarly, we use the following LP relaxation of MKPFG (1.2):

$$
\begin{array}{lll}
\min & \sum_{j \in V} c_{j} x_{j} & \\
\text { s.t. } & \sum_{j \in V \backslash A} a_{j}(A) x_{j} \geq b(A), & \forall A \subseteq V, \\
& x_{i}+x_{j} \geq 1, & \forall\{i, j\} \in E, \\
& x_{j} \geq 0, & \forall j \in V .
\end{array}
$$

The dual of (2.3) is represented as

$$
\begin{array}{lll}
\max & \sum_{A \subseteq V} b(A) y(A)+\sum_{\{i, j\} \in E} z_{\{i, j\}} & \\
\text { s.t. } & \sum_{A \subseteq V: j \notin A} a_{j}(A) y(A)+\sum_{k:\{j, k\} \in E} z_{\{j, k\}} \leq c_{j}, & \forall j \in V, \\
& y(A) \geq 0, & \forall A \subseteq V, \\
& z_{\{i, j\}} \geq 0, & \forall\{i, j\} \in E,
\end{array}
$$

where each dual variable $y(A)$ corresponds to the inequality $\sum_{j \in V \backslash A} a_{j}(A) x_{j} \geq b(A)$ and $z_{\{i, j\}}$ corresponds to the forcing constraint for the edge $\{i, j\}$.

Now we introduce a well-known result for a primal-dual pair of linear programming [6]. Lemma 2.1. Let $\overline{\boldsymbol{x}}$ and $\overline{\boldsymbol{y}}$ be feasible solutions for the following primal and dual linear programming problems:

$$
\min \left\{\boldsymbol{c}^{T} \boldsymbol{x} \mid \boldsymbol{A} \boldsymbol{x} \geq \boldsymbol{b}, \boldsymbol{x} \geq \mathbf{0}\right\} \quad \text { and } \max \left\{\boldsymbol{b}^{T} \boldsymbol{y} \mid \boldsymbol{A}^{T} \boldsymbol{y} \leq \boldsymbol{c}, \boldsymbol{y} \geq \mathbf{0}\right\} .
$$

If the conditions

$$
\begin{aligned}
& \text { (a): } \forall j \in\{1, \cdots, n\}, \bar{x}_{j}>0 \Rightarrow \sum_{i=1}^{m} a_{i j} \bar{y}_{i}=c_{j}, \\
& \text { (b): } \forall i \in\{1, \cdots, m\}, \bar{y}_{i}>0 \Rightarrow \sum_{j=1}^{n} a_{i j} \bar{x}_{j} \leq \alpha b_{i}
\end{aligned}
$$

hold, then $\overline{\boldsymbol{x}}$ is a solution within a factor of $\alpha$ of the optimal solution, that is, the primal objective value $\boldsymbol{c}^{T} \overline{\boldsymbol{x}}$ is less than or equal to a times the optimal value. (Note that the primal problem has an optimal solution because both the primal and dual problems are feasible.).

By applying Lemma 2.1 to the problems (2.3) and (2.4), we have the following lemma and corollary.

Lemma 2.2. Let $\boldsymbol{x}$ and $(\boldsymbol{y}, \boldsymbol{z})$ be feasible solutions for (2.3) and (2.4), respectively. If these solutions satisfy

$$
\begin{array}{ll}
\text { (a): } & \forall j \in V, x_{j}>0 \Rightarrow \sum_{A \subseteq V: j \notin A} a_{j}(A) y(A)+\sum_{k:\{j, k\} \in E} z_{\{j, k\}}=c_{j}, \\
\text { (b-1): } & \forall\{i, j\} \in E, z_{\{i, j\}}>0 \Rightarrow x_{i}+x_{j} \leq 2, \\
\text { (b-2): } & \forall A \subseteq V, y(A)>0 \Rightarrow \sum_{j \in V \backslash A} a_{j}(A) x_{j} \leq 2 b(A),
\end{array}
$$

then $\boldsymbol{x}$ is a solution within a factor of 2 of the optimal solution of (2.3).

Corollary 2.1. Let $\boldsymbol{x}$ be a feasible 0-1 solution of (2.3) and $(\boldsymbol{y}, \boldsymbol{z})$ be a feasible solution of (2.4). If these solutions satisfy (2.5), $\boldsymbol{x}$ is a solution within a factor of 2 of the optimal solution of (1.2).

We propose a polynomial algorithm for calculating $\boldsymbol{x}$ and $(\boldsymbol{y}, \boldsymbol{z})$ which satisfy the conditions in Corollary 2.1. The algorithm generates a sequence of points $\boldsymbol{x}$ and $(\boldsymbol{y}, \boldsymbol{z})$ which always satisfy the following conditions: 
- $\boldsymbol{x} \in\{0,1\}^{n}$.

- $(\boldsymbol{y}, \boldsymbol{z})$ is feasible for $(2.4)$.

- $\boldsymbol{x}$ and $(\boldsymbol{y}, \boldsymbol{z})$ satisfy $(2.5)$.

All the forcing constraints in (2.3) are satisfied in Step 1 and the other constraints in (2.3) are met in Step 2. For the points $\boldsymbol{x}$ and $(\boldsymbol{y}, \boldsymbol{z})$ at each step, we use symbols $S=\left\{j \in V \mid x_{j}=1\right\}$, $\bar{b}=b-\sum_{j \in V} a_{j} x_{j}$, and $\overline{c_{j}}=c_{j}-\left(\sum_{A \subseteq V: j \notin A} a_{j}(A) y(A)+\sum_{k:\{j, k\} \in E} z_{\{j, k\}}\right)$ for $j \in V . \bar{E} \subseteq E$ denotes a set of unchecked edges in Step 1. Now we state our algorithm.

\section{Algorithm 1}

Input: $G=(V, E), a_{j}, c_{j}(j \in V)$ and $b$.

Output: $\tilde{\boldsymbol{x}}$ and $(\tilde{\boldsymbol{y}}, \tilde{\boldsymbol{z}})$.

Step 0: Let $\boldsymbol{x}=\mathbf{0}$ and $(\boldsymbol{y}, \boldsymbol{z})=(\mathbf{0}, \mathbf{0})$ be initial solutions. Set $S=\emptyset, V^{\prime}=\left\{j \in V \mid a_{j}>\right.$ $0\}, \bar{E}=E, \bar{b}=b$, and $\bar{c}_{j}=c_{j}$ for $j \in V$.

Step 1: If $\bar{E}=\emptyset$, then go to Step 2. Otherwise choose an edge $e=\{i, j\} \in \bar{E}$. If $x_{i}+x_{j} \geq 1$, then update $\bar{E}=\bar{E} \backslash\{e\}$ and go back to the top of Step 1. If $x_{i}+x_{j}=0$, increase $z_{\{i, j\}}$ as much as possible while maintaining feasibility for (2.4). Since $z_{\{i, j\}}$ appears in only two constraints of (2.4) corresponding to the vertices $i$ and $j$, we see that

$$
z_{\{i, j\}}=\bar{c}_{s} \quad \text { for } \quad s=\arg \min \left\{\bar{c}_{i}, \bar{c}_{j}\right\} .
$$

Update $x_{s}=1, S=S \cup\{s\}, \bar{E}=\bar{E} \backslash\{e\}, \bar{c}_{i}=\bar{c}_{i}-z_{\{i, j\}}, \bar{c}_{j}=\bar{c}_{j}-z_{\{i, j\}}$, and $\bar{b}=\bar{b}-a_{s}$. Go back to the top of Step 1.

Step 2: If $\bar{b} \leq 0$, then output $\tilde{\boldsymbol{x}}=\boldsymbol{x}$ and $(\tilde{\boldsymbol{y}}, \tilde{\boldsymbol{z}})=(\boldsymbol{y}, \boldsymbol{z})$ and stop. Otherwise calculate $a_{j}(S)$ for all $j \in V^{\prime} \backslash S$ by $(2.2)$, where $b(S)=\bar{b}$. Increase $y(S)$ as much as possible while maintaining feasibility for (2.4). Since $y(S)$ appears in constraints of (2.4) for $j \in V^{\prime} \backslash S$ so that $a_{j}(S)>0$, we see that

$$
y(S)=\frac{\bar{c}_{s}}{a_{s}(S)} \quad \text { for } \quad s=\arg \min _{j \in V^{\prime} \backslash S}\left\{\frac{\bar{c}_{j}}{a_{j}(S)}\right\} .
$$

Update $x_{s}=1, S=S \cup\{s\}, \bar{c}_{j}=\bar{c}_{j}-a_{j}(S) y(S)$ for any $j \in V^{\prime} \backslash S$, and $\bar{b}=\bar{b}-a_{s}$. Go back to the top of Step 2.

For the outputs $\tilde{\boldsymbol{x}}$ and $(\tilde{\boldsymbol{y}}, \tilde{\boldsymbol{z}})$ of Algorithm 1, we have the following results.

Lemma 2.3. $\tilde{\boldsymbol{x}}$ is a feasible 0-1 solution of (2.3) and $(\tilde{\boldsymbol{y}}, \tilde{\boldsymbol{z}})$ is a feasible solution of (2.4).

Proof. By the assumption that MKPFG (1.2) is feasible, $\boldsymbol{x}=(1, \cdots, 1)$ is feasible for the LP relaxation problem (2.3). Algorithm 1 starts from $\boldsymbol{x}=\mathbf{0}$ and updates a variable $x_{j}$ from 0 to 1 at each iteration until all the constraints in (2.3) are satisfied. Hence $\tilde{\boldsymbol{x}}$ is a feasible $0-1$ solution of $(2.3)$.

Algorithm 1 starts from the dual feasible solution $(\boldsymbol{y}, \boldsymbol{z})=(\mathbf{0}, \mathbf{0})$ and maintains dual feasibility throughout the algorithm. Hence $(\tilde{\boldsymbol{y}}, \tilde{\boldsymbol{z}})$ is feasible for $(2.4)$.

Lemma 2.4. $\tilde{\boldsymbol{x}}$ and $(\tilde{\boldsymbol{y}}, \tilde{\boldsymbol{z}})$ satisfy (2.5).

Proof. Since $\boldsymbol{x}=\mathbf{0}$ at the beginning and the algorithm sets $x_{j}=1$ only if the $j$-th constraint in (2.4) becomes tight, (a) of (2.5) is satisfied. (b-1) of (2.5) follows from $\tilde{\boldsymbol{x}} \in\{0,1\}^{n}$. Thus 
it suffices to show that (b-2) holds. We consider two cases, whether or not the algorithm stops at the first iteration of Step 2.

If the algorithm stops at the first iteration of Step 2, we obtain a primal feasible solution in Step 1. Then (b-2) holds since $\tilde{y}(A)=0$ for any $A \subseteq V$. Conversely, if we have a primal feasible solution in Step 1, then the algorithm stops at the first iteration of Step 2 since $\bar{b} \leq 0$ holds.

Suppose that the algorithm does not stop at the first iteration of Step 2. Define $\tilde{S}=$ $\left\{j \in V \mid \tilde{x}_{j}=1\right\}$. Let $\tilde{x}_{\ell}$ be the variable which becomes 1 from 0 at the last iteration of Step 2. From Step 2, $\tilde{y}(A)>0$ implies

$$
A \subseteq \tilde{S} \backslash\{\ell\}
$$

Since the algorithm does not stop just before setting $\tilde{x}_{\ell}=1$, we have

$$
\sum_{j \in \tilde{S} \backslash\{\ell\}} a_{j}<b
$$

By (2.6) and (2.7), we observe that for any subset $A \subseteq N$ such that $\tilde{y}(A)>0$

$$
\sum_{j \in(\tilde{S} \backslash\{\ell\}) \backslash A} a_{j}(A) \leq \sum_{j \in(\tilde{S} \backslash\{\ell\}) \backslash A} a_{j}=\sum_{j \in \tilde{S} \backslash\{\ell\}} a_{j}-\sum_{j \in A} a_{j}<b-\sum_{j \in A} a_{j} \leq b(A),
$$

where the first and last inequality follows from the definitions $(2.2)$ of $a_{j}(A)$ and $b(A)$. Thus, we have that for any subset $A \subseteq N$ such that $\tilde{y}(A)>0$

$$
\sum_{j \in V \backslash A} a_{j}(A) \tilde{x}_{j}=\sum_{j \in \tilde{S} \backslash A} a_{j}(A)=\sum_{j \in(\tilde{S} \backslash\{\ell\}) \backslash A} a_{j}(A)+a_{\ell}(A)<2 b(A),
$$

where the last inequality follows from $a_{\ell}(A) \leq b(A)$.

Lemma 2.5. The running time of Algorithm 1 is $O\left(|E|+\left|V^{\prime}\right|^{2}\right)$, where $V^{\prime}=\left\{j \in V \mid a_{j}>\right.$ $0\}$.

Proof. The running time of one iteration of Step 1 is $O(1)$ and the number of iterations in Step 1 is at most $|E|$. The running time of one iteration of Step 2 is $O\left(\left|V^{\prime}\right|\right)$ and the number of iterations in Step 2 is at most $\left|V^{\prime}\right|$. Therefore the running time of the algorithm is $O\left(|E|+\left|V^{\prime}\right|^{2}\right)$.

The following result follows from Corollary 2.1 and Lemmas 2.3, 2.4, and 2.5.

Theorem 2.1. Algorithm 1 is a 2-approximation algorithm for MKPFG (1.2).

\section{Generalization to a Covering Integer Program with 0-1 Variables}

In this section, we generalize Algorithm 1 to a covering integer program with $0-1$ variables (CIP), which is represented as

$$
\begin{array}{l|ll}
\operatorname{CIP} & \min & \sum_{j \in N} c_{j} x_{j} \\
\text { s.t. } & \sum_{j \in N} a_{i j} x_{j} \geq b_{i}, \quad \forall i \in M=\{1, \cdots, m\}, \\
& x_{j} \in\{0,1\}, \quad \forall j \in N=\{1, \cdots, n\},
\end{array}
$$


where $b_{i}, a_{i j}$, and $c_{j}(i \in M, j \in N)$ are nonnegative. Assume that $\sum_{j \in N} a_{i j} \geq b_{i}$ for any $i \in M$, so that the problem is feasible. Let $\Delta_{i}$ be the number of non-zero coefficients in the $i$-th constraint $\sum_{j \in N} a_{i j} x_{j} \geq b_{i}$. Without loss of generality, we assume that $\Delta_{1} \geq$ $\Delta_{2} \geq \cdots \geq \Delta_{m}$ and $\Delta_{2} \geq 2$. There are some $\Delta_{1}$-approximation algorithms for CIP, see Koufogiannakis and Young [10] and references therein. We propose a $\Delta_{2}$-approximation algorithm. The minimum knapsack problem with a forcing graph (1.2) is a special case of CIP for which $\Delta_{2}=2$.

We introduce an LP relaxation problem of CIP constructed by Carr et al. [3]. The relaxation problem is represented as

$$
\begin{array}{lll}
\min & \sum_{j \in N} c_{j} x_{j} & \\
\text { s.t. } & \sum_{j \in N \backslash A} a_{i j}(A) x_{j} \geq b_{i}(A), \quad \forall A \subseteq N, \quad \forall i \in M, \\
& x_{j} \geq 0, & \forall j \in N,
\end{array}
$$

where

$$
\begin{aligned}
b_{i}(A) & =\max \left\{0, b_{i}-\sum_{j \in A} a_{i j}\right\}, \forall i \in M, \forall A \subseteq N, \\
a_{i j}(A) & =\min \left\{a_{i j}, b_{i}(A)\right\}, \forall i \in M, \forall A \subseteq N, \forall j \in N \backslash A .
\end{aligned}
$$

Carr et al. [3] show that any feasible $0-1$ solution of (3.2) is feasible for (3.1). The dual problem of (3.2) can be stated as

$$
\begin{array}{lll}
\max & \sum_{i \in M} \sum_{A \subseteq N} b_{i}(A) y_{i}(A) & \\
\text { s.t. } & \sum_{i \in M} \sum_{A \subseteq N: j \notin A} a_{i j}(A) y_{i}(A) \leq c_{j}, \quad \forall j \in N, & \\
& y_{i}(A) \geq 0, & \forall A \subseteq N, \forall i \in M .
\end{array}
$$

By applying Lemma 2.1 to the LP problems (3.2) and (3.4), we have the following result. Lemma 3.1. Let $\boldsymbol{x}$ be a feasible 0-1 solution of (3.2) and $\boldsymbol{y}$ be a feasible solution of (3.4). If these solutions satisfy

$$
\begin{aligned}
& \text { (a): } \forall j \in N, x_{j}>0 \Rightarrow \sum_{i \in M} \sum_{A \subseteq N: j \notin A} a_{i j}(A) y_{i}(A)=c_{j}, \\
& \text { (b): } \forall i \in M, \forall A \subseteq N, y_{i}(A)>0 \Rightarrow \sum_{j \in N \backslash A} a_{i j}(A) x_{j} \leq \Delta_{2} b(A),
\end{aligned}
$$

then $\boldsymbol{x}$ is a solution within a factor of $\Delta_{2}$ of the optimal solution of (3.1).

Our algorithm is presented in Algorithm 2 below. The goal is to find $\boldsymbol{x}$ and $\boldsymbol{y}$ which satisfy the conditions in Lemma 3.1. The algorithm generates a sequence of points $\boldsymbol{x}$ and $\boldsymbol{y}$. Throughout the algorithm, the conditions $\boldsymbol{x} \in\{0,1\}^{n}$, constraints in (3.4), and (3.5) are satisfied. The constraints in (3.2) are satisfied at Step 2. In Algorithm 2, we use the symbols $S=\left\{j \in N \mid x_{j}=1\right\}, b_{i}(S)=\max \left\{0, b_{i}-\sum_{j \in S} a_{i j}\right\}$ for $i \in M$, and $\overline{c_{j}}=c_{j}-\sum_{i \in M} \sum_{A \subseteq N: j \notin A} a_{i j}(A) y_{i}(A)$ for $j \in N$.

\section{Algorithm 2}

Input: $M, N, a_{i j}, b_{i}$ and $c_{j}(i \in M, j \in N)$.

Output: $\tilde{\boldsymbol{x}}$ and $\tilde{\boldsymbol{y}}$.

Step 0: Set $\boldsymbol{x}=\mathbf{0}, \boldsymbol{y}=\mathbf{0}$, and $S=\emptyset$. Let $N_{i}^{\prime}=\left\{j \in N \mid a_{i j}>0\right\}$ for $i \in M, \bar{c}_{j}=c_{j}$ for $j \in N$, and $i=m$. 
Step 1: If $i=0$, then output $\tilde{\boldsymbol{x}}=\boldsymbol{x}$ and $\tilde{\boldsymbol{y}}=\boldsymbol{y}$ and stop. Otherwise set $b_{i}(S)=\max \left\{0, b_{i}-\right.$ $\left.\sum_{j \in S} a_{i j}\right\}$ and go to Step 2 .

Step 2: If $b_{i}(S)=0$, then update $i=i-1$ and go to Step 1. Otherwise calculate $a_{i j}(S)$ for any $j \in N_{i}^{\prime} \backslash S$ by (3.3). Increase $y_{i}(S)$ while maintaining dual feasibility until at least one constraint $s \in N_{i}^{\prime} \backslash S$ is tight. Namely set

$$
y_{i}(S)=\frac{\bar{c}_{s}}{a_{i s}(S)} \quad \text { for } \quad s=\arg \min _{j \in N_{i}^{\prime} \backslash S}\left\{\frac{\bar{c}_{j}}{a_{i j}(S)}\right\} .
$$

Update $\bar{c}_{j}=\bar{c}_{j}-a_{i j}(S) y_{i}(S)$ for $j \in N^{\prime} \backslash S, x_{s}=1, S=S \cup\{s\}$, and $b_{i}(S)=$ $\max \left\{0, b_{i}(S)-a_{i s}\right\}$. Go back to the top of Step 2 .

In the same way as the proof of Lemma 2.3, we have the following result for the outputs $\tilde{\boldsymbol{x}}$ and $\tilde{\boldsymbol{y}}$ of Algorithm 2.

Lemma 3.2. $\tilde{\boldsymbol{x}}$ is a $0-1$ feasible solution of (3.2) and $\tilde{\boldsymbol{y}}$ is a feasible solution of (3.4).

The next lemma is similarly proved as Lemma 2.4 .

Lemma 3.3. $\tilde{\boldsymbol{x}}$ and $\tilde{\boldsymbol{y}}$ satisfy (3.5).

Proof. All the conditions in (a) of (3.5) are naturally satisfied by the way the algorithm updates primal variables. It suffices to show that all the conditions in (b) are satisfied. For any $i \in\{2, \cdots, m\}$ and any subset $A \subseteq N$ such that $\tilde{y}_{i}(A)>0$, we obtain that

$$
\sum_{j \in N \backslash A} a_{i j}(A) \tilde{x}_{j} \leq \Delta_{i} b_{i}(A) \leq \Delta_{2} b_{i}(A)
$$

since $a_{i j}(A) \leq b_{i}(A)$ by the definition (3.3) and the $i$-th constraint has $\Delta_{i}$ non-zero coefficients. Then, we consider the case of $i=1$. In a similar way of the proof in Lemma 2.4, for any subset $A \subseteq N$ such that $\tilde{y}_{1}(A)>0$, we have

$$
\sum_{j \in N \backslash A} a_{1 j}(A) \tilde{x}_{j} \leq 2 b_{1}(A) \leq \Delta_{2} b_{1}(A) .
$$

Lemma 3.4. The running time of Algorithm 2 is $O\left(\Delta_{1}(m+n)\right)$.

Proof. The running time of one iteration of Step 1 is $O\left(\Delta_{1}\right)$ and the number of iterations in Step 1 is at most $m$. On the other hand, the running time of one iteration of Step 2 is $O\left(\Delta_{1}\right)$ and the number of iterations in Step 2 is at most $m+n$. Therefore the total running time of the algorithm is $O\left(\Delta_{1} m\right)+O\left(\Delta_{1}(m+n)\right)=O\left(\Delta_{1}(m+n)\right)$.

From the results above, we can obtain the next theorem.

Theorem 3.1. Algorithm 2 is a $\Delta_{2}$-approximation algorithm for CIP (3.1).

\section{Conclusion}

We proposed a 2-approximation algorithm for the minimum knapsack problem with a forcing graph. The performance ratio of the algorithm is the same as that of the algorithms for the minimum knapsack problem presented by Carnes and Shmoys [2] and for the minimum vertex cover problem by Bar-Yehuda and Even [1]. Then we generalize the algorithm to the covering integer program with 0 - 1 variables and proposed a $\Delta_{2}$-approximation algorithm, where $\Delta_{2}$ is the second largest number of non-zero coefficients in the constraints. 


\section{Acknowledgment}

The authors are grateful to anonymous reviewers for their careful reading and many helpful comments. This research is supported in part by Grant-in-Aid for Science Research (A) 26242027 of Japan Society for the Promotion of Science.

\section{References}

[1] R. Bar-Yehuda and S. Even: A linear-time approximation algorithm for the weighted vertex cover problem. Journal of Algorithms, 2 (1981), 198-203.

[2] T. Carnes and D. Shmoys: Primal-dual schema for capacitated covering problems. Mathematical Programming, 153 (2015), 289-308.

[3] R.D. Carr, L. Fleischer, V.J. Leung, and C.A. Phillips: Strengthening integrality gaps for capacitated network design and covering problems. Proceedings of the 11th Annual ACM-SIAM Symposium on Discrete Algorithms (2000), 106-115.

[4] A. Darmann, U. Pferschy, J. Schauer, and G.J. Woeginger: Paths, trees and matchings under disjunctive constraints. Discrete Applied Mathematics, 159 (2011), 1726-1735.

[5] I. Dinur and S. Safra: On the hardness of approximating minimum vertex cover. Annals of Mathematics, 162 (2005), 439-485.

[6] D. Du, K. Ko, and X. Hu: Design and Analysis of Approximation Algorithms (SpringerVerlag, 2011), 297-303.

[7] M. Hifi and N. Otmani: An algorithm for the disjunctively constrained knapsack problem. International Journal of Operational Research, 13 (2012), 22-43.

[8] M. Hifi, S. Saleh, and L. Wu: A fast large neighborhood search for disjunctively constrained knapsack problems. Proceedings of 3rd International Symposium on Combinatorial Optimization, ISCO2014, volume 8596 of Lecture Notes in Computer Science (Springer, 2014), 396-407.

[9] S. Khot and O. Regev: Vertex cover might be hard to approximate to within 2- $\epsilon$. Journal of Computer and System Sciences, 74 (2008), 335-349.

[10] C. Koufogiannakis and N.E. Young: Greedy $\delta$-approximation algorithm for covering with arbitrary constraints and submodular cost. Algorithmica, 66 (2013), 113-152.

[11] U. Pferschy and J. Schauer: The knapsack problem with conflict graphs. Journal of Graph Algorithms and Applications, 13 (2009), 233-249.

[12] U. Pferschy and J. Schauer: The maximum flow problem with disjunctive constraints. Journal of Combinatorial Optimization, 26 (2013), 109-119.

[13] U. Pferschy and J. Schauer: Approximation of knapsack problems with conflict and forcing graphs. to appear in Journal of Combinatorial Optimization (2016).

[14] C. Wilbaut, S. Hanafi, and S. Salhi: A survey of effective heuristics and their applications to a variety of knapsack problems. IMA Journal of Management Mathematics, 19 (2008), 227-244.

[15] T. Yamada, S. Kataoka, and K. Watanabe: Heuristic and exact algorithms for the disjunctively constrained knapsack problem. Information Processing Society of Japan Journal, 43 (2002), 2864-2870. 
Yotaro Takazawa

Department of Industrial Engineering and Management

Tokyo Institute of Technology

2-12-1 Ohokayama

Meguro-ku Tokyo 152-8552, Japan

E-mail: takazawa.y.ab@m.titech.ac.jp 